\title{
Implementation Challenges for Multivariable Control \\ What You Did Not Learn in School!
}

\section{Sanjay Garg}

Glenn Research Center, Cleveland, Ohio 


\section{NASA STI Program . . . in Profile}

Since its founding, NASA has been dedicated to the advancement of aeronautics and space science. The NASA Scientific and Technical Information (STI) program plays a key part in helping NASA maintain this important role.

The NASA STI Program operates under the auspices of the Agency Chief Information Officer. It collects, organizes, provides for archiving, and disseminates NASA's STI. The NASA STI program provides access to the NASA Aeronautics and Space Database and its public interface, the NASA Technical Reports Server, thus providing one of the largest collections of aeronautical and space science STI in the world. Results are published in both non-NASA channels and by NASA in the NASA STI Report Series, which includes the following report types:

- TECHNICAL PUBLICATION. Reports of completed research or a major significant phase of research that present the results of NASA programs and include extensive data or theoretical analysis. Includes compilations of significant scientific and technical data and information deemed to be of continuing reference value. NASA counterpart of peer-reviewed formal professional papers but has less stringent limitations on manuscript length and extent of graphic presentations.

- TECHNICAL MEMORANDUM. Scientific and technical findings that are preliminary or of specialized interest, e.g., quick release reports, working papers, and bibliographies that contain minimal annotation. Does not contain extensive analysis.

- CONTRACTOR REPORT. Scientific and technical findings by NASA-sponsored contractors and grantees.

- CONFERENCE PUBLICATION. Collected papers from scientific and technical conferences, symposia, seminars, or other meetings sponsored or cosponsored by NASA.

- SPECIAL PUBLICATION. Scientific, technical, or historical information from NASA programs, projects, and missions, often concerned with subjects having substantial public interest.

- TECHNICAL TRANSLATION. Englishlanguage translations of foreign scientific and technical material pertinent to NASA's mission.

Specialized services also include creating custom thesauri, building customized databases, organizing and publishing research results.

For more information about the NASA STI program, see the following:

- Access the NASA STI program home page at http://www.sti.nasa.gov

- E-mail your question via the Internet to help@ sti.nasa.gov

- Fax your question to the NASA STI Help Desk at 301-621-0134

- Telephone the NASA STI Help Desk at 301-621-0390

- Write to: NASA Center for AeroSpace Information (CASI) 7115 Standard Drive Hanover, MD 21076-1320 


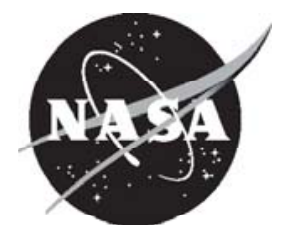

\section{Implementation Challenges for Multivariable Control \\ What You Did Not Learn in School!}

\section{Sanjay Garg}

Glenn Research Center, Cleveland, Ohio

Prepared for the

Guidance, Navigation and Control Conference

sponsored by the American Institute of Aeronautics and Astronautics

Hilton Head, South Carolina, August 20-23, 2007

National Aeronautics and

Space Administration

Glenn Research Center

Cleveland, Ohio 44135 
Level of Review: This material has been technically reviewed by technical management.

Available from

NASA Center for Aerospace Information 7115 Standard Drive

Hanover, MD 21076-1320
National Technical Information Service 5285 Port Royal Road Springfield, VA 22161

Available electronically at http://gltrs.grc.nasa.gov 


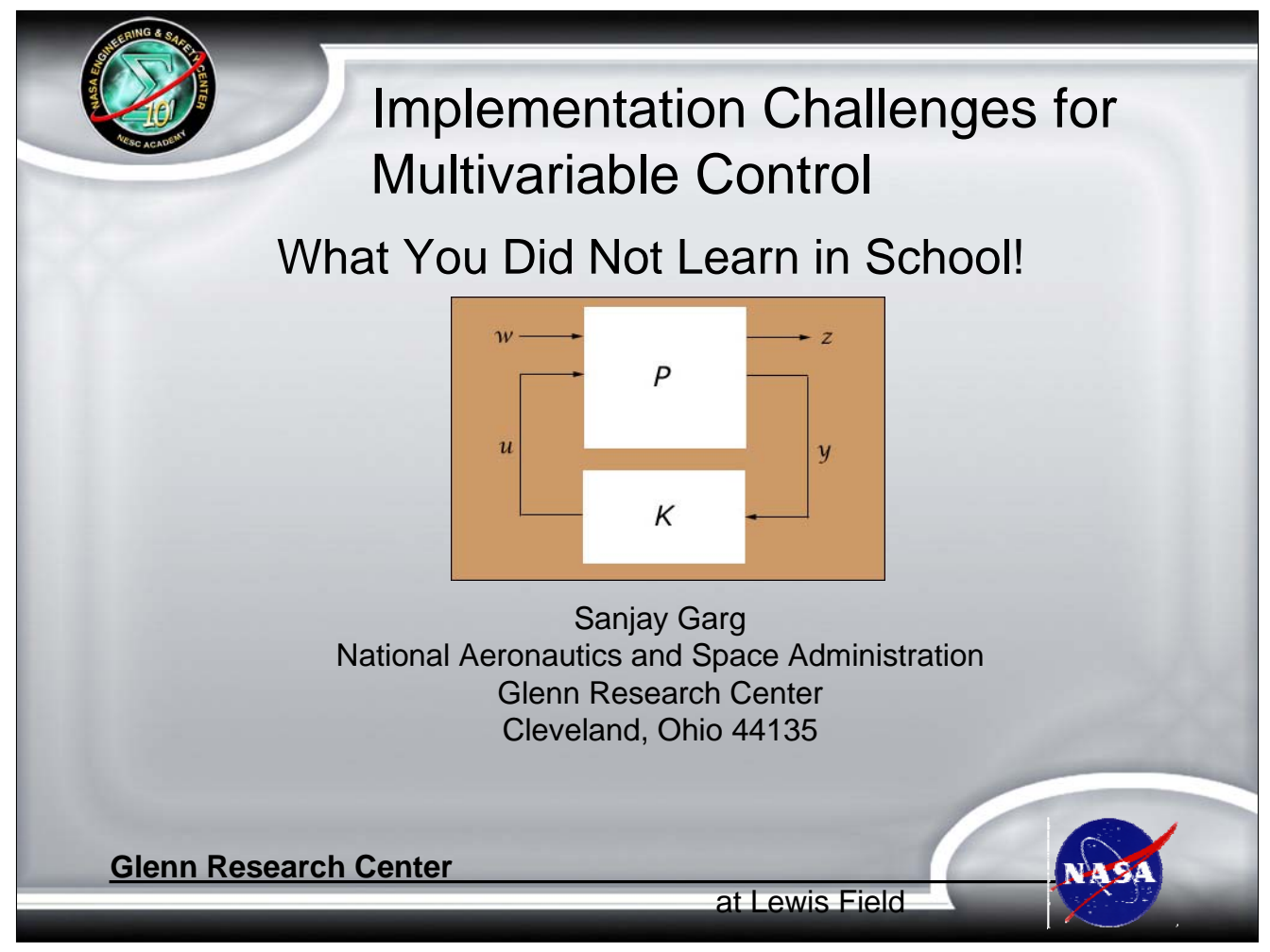

\begin{abstract}
Multivariable control allows controller designs that can provide decoupled command tracking and robust performance in the presence of modeling uncertainties. Although the last two decades have seen extensive development of multivariable control theory and example applications to complex systems in software/hardware simulations, there are no "production" flying systems - aircraft or spacecraft, that use multivariable control. This is because of the tremendous challenges associated with implementation of such multivariable control designs. Unfortunately, the curriculum in schools does not provide sufficient time to be able to provide an exposure to the students in such implementation challenges. The objective of this paper is to share the lessons learned by a practitioner of multivariable control in the process of applying some of the modern control theory to the Integrated Flight Propulsion Control (IFPC) design for an advanced Short Take-Off Vertical Landing (STOVL) aircraft simulation.
\end{abstract}




\section{Overview}

- Control design problem and typical mathematical formulation

- Unique nature of multivariable control (MVC)

- Benefits and Capabilities of MVC

- Challenges and barriers to using MVC in real systems (with reference to STOVL IFPC Design)

- Right problem formulation

- Major implementation issues

- Making changes on-the-fly

- Conclusion

Glenn Research Center at Lewis_Field

\section{Overview}

The paper, which is based on a lecture on the topic as part of the NASA NESC (NASA Engineering and Safety Center) course on Satellite Attitude Control Systems, covers the following topics: i) Unique nature of "Multivariable Control (MVC)"; ii) Benefits and Capabilities of Multivariable Control; iii) Challenges and barriers of using multivariable control designs in real flight systems. The paper starts by describing what is meant by Multivariable Control and provides a brief comparison of "Classical" versus "Modern" methods of control law design. The motivation for using Multivariable Control is then discussed in terms of robustness and performance benefits of various synthesis approaches (e.g., LQG/LTR (Linear Quadratic Gaussian/Loop Transfer Recovery), H-infinity etc.). A practitioner's perspective is provided on when to use Multivariable Control - and more importantly when not to! Some of the challenges associated with formulating the problem right within the limitations and flexibilities of the multivariable control synthesis techniques are then discussed with a focus on 3 areas: Control effector blending, addressing nonlinearities, and controller stability. Insight is provided into two major issues with implementing multivariable control: Gain Scheduling, and Integrator Wind up Protection. Finally, the issue of having to make amendments based on problems encountered during testing and/or operation is discussed.

The above challenges and barriers are discussed with reference to the author's experience with the STOVL IFPC design and its evaluation on a fixed-base piloted simulation. Tools and techniques for overcoming some of the barriers are also presented with illustrative examples from the STOVL IFPC design study. The hope is that this paper will provide a basis for students of multivariable control theory to get a broad perspective of issues that need to be addressed when it comes to implementing the theory based designs to complex systems. 


\section{Control Design Problem}

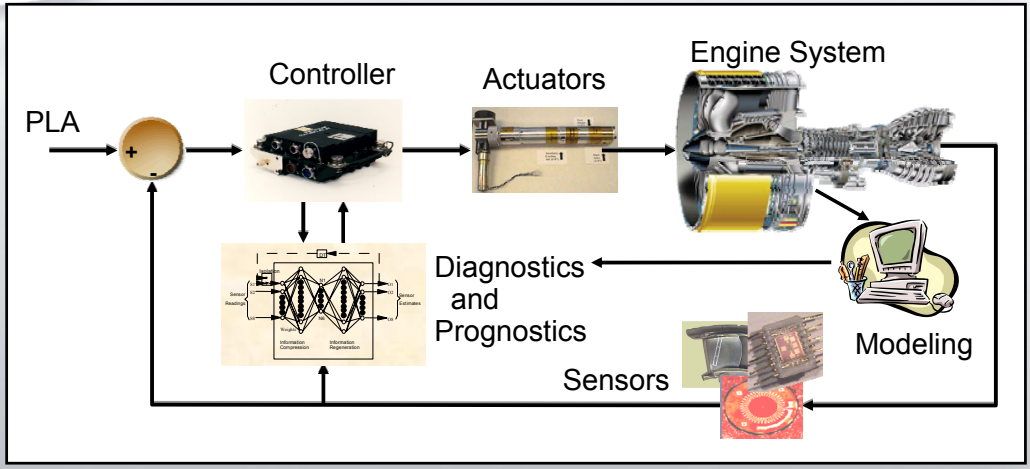

- Given model of "plant," find control law which provides:

- Desired command tracking in presence of disturbances

- Robustness to modeling uncertainties

- Stable operation when encountering limits

- Keep integrated system perspective when developing control

Glenn Research Center

atLewis_Field

\section{Control Design Problem}

Simply stated, the control design problem is: Given a model of the "plant", find control law which provides desired command tracking in the presence of disturbances, robustness to modeling uncertainties and stable operation when encountering limits. Although the focus of this paper is on the control laws which are embedded in the controller hardware, it is important to keep an overall systems perspective in mind when designing the control laws since a control system consists of many separate components, such as the actual control hardware and software, an actuation system, a sensing system, on-board models etc.

It is important to note that the real plant always has some disturbances e.g., gust for flight control. These disturbances need to be adequately represented in the formulation of the control design problem. Also, do not forget, that when you do the control law design, you're working with a mathematical model of the plant. Modeling has many issues; there are uncertainties associated with the modeling approach as well as the parameters used to represent physical phenomenon, approximations are made to keep the mathematical model tractable, etc. It is important to be knowledgeable about the assumptions made in developing the mathematical model of the plant and what impact these assumptions might have in terms of controlling the model vs. controlling the plant. The importance of robustness to modeling uncertainties is very well understood and there has been extensive literature on robust control design methods.

However, the third requirement, to have stable operation when encountering limits, is typically not covered in control courses. Every system has hardware limits, such as actuator positions and rates, as well as operational limits imposed for safety. It is important to have a good understanding of the typical limits for a system and to build in limit protection such that the control laws will provide graceful performance degradation and will not drive the system unstable when limits are encountered. 


\section{MVC Unique Nature}

- Systems with $>1$ output being controlled by $>1$ manipulated input, with any given input affecting more than single output: multiinput/multi-output (MIMO)

- "Classical" methods: based primarily on frequency domain approach, extend single-input/single-output (SISO) transfer function techniques, e.g., Nyquist, to MIMO transfer function matrix

- "Modern" methods: based on state-space approach, formulation of quadratic cost function resulting in linear control law typically solved through system of Ricatti equations

- Control solution easily amenable for digital implementation

- Analysis typically done using multivariable frequency domain techniques

- Unlike simple gain and phase margin measures for SISO, "robustness" determination of MVC control requires complex analyses using singular value techniques and Monte Carlo simulations

\section{MVC Unique Nature}

A multivariable control or a multi-input/multi-output (MIMO) system is a system with more than one output being controlled by more than one manipulated input. And the important part is that any given input affects more than a single output. If each input only affects a single output, then you basically have a combination of single-input/single-output systems, and you don't need multivariable control for that. There are many approaches to MVC - some approaches such as Multivariable Nyquist Theory extend the classical single-input/single-output approaches to multivariable control, modern state-space based methods which center around an optimal solution of a quadratic cost function, optimization methods such as neural networks etc. The discussion in the rest of the paper is pertinent to any multivariable control design regardless of the synthesis approach, however, the discussion will be based on controllers synthesized using modern state-space based methods. There are emerging nonlinear control design approaches such as MPC (Model Predictive Control) which might not have some of the same issues as those being discussed in this paper. However, such approaches are still at a development stage with respect to aerospace applications.

As an aside, it is important to note that the quadratic formulation of the cost function is used in typical state-space methods, such as LQR (Linear Quadratic Regulator) because it results in an optimal control law which is linear - typically obtained through solving a system of Ricatti equations. The control solution is easily amenable to digital implementation, as well as allows all the linear analysis tools to be used for performance and robustness analysis of the closed-loop system.

Analysis of control laws is still done using frequency domain techniques. So, to be a good control design engineer, it is very important to get the basics right with respect to frequency domain analyses. If you don't understand gain margin, phase margin, Bode plots etc. and their implications for closed loop system performance and robustness, it's very, very difficult to apply the multivariable frequency domain analyses techniques, such as singular value analysis and "mu-analysis" techniques in a meaningful way. 


\section{MVC Benefits and Capabilities}

- "1-step" control solution for complex intercoupled systems

- Decoupled command tracking

- "Optimal" control usage

- When to use MVC:

- System has high level of input/output coupling and not much separation in loop bandwidths

- Physics of problem are reasonably well-understood to be able to use "design handles" effectively

- System is complex with large number of states

- When sequential SISO loop closure will not meet performance requirements!

- Remember: MVC cannot make 747 fly like F-16 !

Glenn Research Center at Lewis Field

\section{MVC Benefits and Capabilities}

Why use multivariable control? Because if you have a truly MIMO (multi-input/multi-output) system, doing sequential loop closures or trying to do decoupled command tracking becomes very, very difficult. Multivariable control provides "one-step" solution for complex intercoupled systems with systematic approaches to perform the fundamental trade-offs between performance, control usage and robustness. Although the typical quadratic cost function formulation implies that the control law is "optimal" in some way, as will be discussed later, it is important to be aware that the "optimality" is based on the way the design plant is formulated, including the choice of the weights in the quadratic function, for control law synthesis and it might lead to unexpected results in terms of control usage when implemented on the system.

Also it is important to emphasize that MVC cannot be meaningfully applied unless the control designer has a good basic understanding of the physics of the system being controlled or works in close coordination with someone who has such expert knowledge. The idea of "give me a math model and give me requirements, and I will come up with a control law that meets the requirements" is fine for a classroom project, but does not work too well in real life. Understanding the physics of the problem allows the control designer to better apply the "design handles" in the selected control synthesis approach and to ensure that the limitations of the math model will not result in a control law that does not meet the design requirements when integrated with the physical system.

No matter how fancy your multivariable control synthesis approach is, or how good a control designer you are, you cannot make a 747 actually fly like an F-16, because of the inherent dynamics of the system and the limitations on the available control authority! The physical properties such as mass, inertia, aerodynamics, control surface sizes etc. determine the limitations on achievable performance, regardless of what control synthesis technique is used. 


\section{Typical MVC Approaches}

- Loop shaping techniques such as LQG/LTR, H-infinity, Mu-synthesis

- Trade-off between performance, robustness and control usage

- Control objectives are indirectly reflected in formulation-requiring iteration to ensure objectives are met

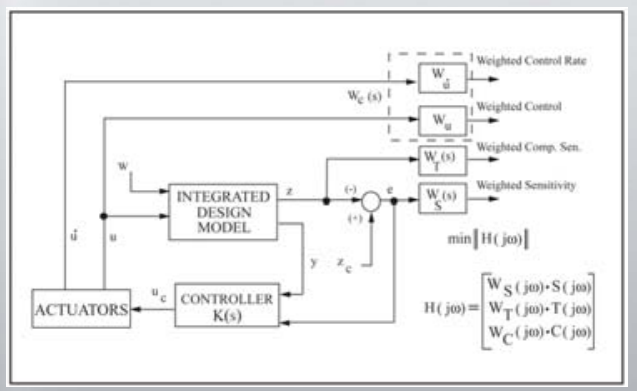

- Critical to formulate problem such that it reflects design goals

- Control rate weighting to reflect rate limits

- Scaling to accommodate large variations in dimensions

-Typical state-based approaches have controller order $\geq$ "plant" order; controller order reduction becomes necessary

Glenn Research Center at Lewis_Field Nos

\section{Typical MVC Approaches}

Modern multivariable control approaches such as H-infinity, mu-synthesis, LQG/LTR (Linear Quadratic Gaussian/Loop Transfer Recovery) take the best of state-space and frequency domain techniques and merge them for robust control synthesis. All these are based on providing "handles" to make intelligent tradeoffs, through trial and error, between performance, robustness and control usage. The fundamental trade-off between performance and robustness for closed-loop systems is evident from the relationship between the sensitivity and transmission (complimentary sensitivity) functions which is covered in a typical multivariable control course.

The generic formulation for $\mathrm{H}$-infinity control synthesis is shown in the block diagram. By selecting appropriate weightings WS, WT, and WC on the sensitivity, transmission and control functions, respectively, the designer can achieve the desired trade-off between performance, robustness and control usage. In terms of control usage, it is important to provide a mechanism to limit the control rate in the synthesis process. If the control rate is not weighted properly, it is possible that the control law will result in large control rates thus hitting the control rate limit and violating all assumptions of linearity. When formulating the design plant, it is also important to keep in mind that scaling has a significant impact on the resulting control law. All the multivariable analyses tools are based on inputs and outputs being of comparable dimensions. For instance, in aircraft engine control, we have interest in controlling the rotor speed, measured in 1000s of rpm, and pressure gain across the fan, measured in single digit psi. If the design model is not scaled appropriately, the large units for fan speed will end up overshadowing the small changes in pressure.

The order of the controller resulting from modern multivariable control methods is equal to or greater than the order of the design plant. From implementation perspective (limited throughput and memory in control hardware), it is important to keep the controller order low, so typically controller order reduction will be necessary. 


\section{MVC Design Challenges: Right Problem Formulation}

- Synthesis methods based on mathematical formulation that allows for "easier" solution-only indirectly address control objectives

- Numerical stability might require some modifications:

- Scaling (discussed earlier)

- Replacing pure integrators with "slow poles"

- Overlooking key physics of problem in process of formulating "design plant" can lead to problems

- Control effector blending

- Addressing nonlinearities

- Controller stability

\section{Glenn Research Center} at Lewis Field

\section{MVC Design Challenges: Right Problem Formulation}

The control synthesis methods are based on a mathematical formulation that allows for "easier" solution - they only indirectly reflect the control objectives. For instance, as discussed earlier, there is no direct way of incorporating control position and rate limits in the synthesis process. Since, typically the control law solution consists of solving a set of algebraic Ricatti equations, it might be necessary to make some modifications in the "design" plant to make sure that numerical stability problems are not encountered. Plant scaling and replacing pure integrators with slow poles are some examples of such modifications.

As was discussed earlier, lack of understanding of the "physics" of the problem can lead to totally unacceptable control law designs. Three things to keep in mind, which have a significant impact on practical applicability of the control law synthesized through the multivariable control design technique are: i) Control effector blending; ii) Addressing nonlinearities within the capability of the linear methods; iii) Assessing the stability of the controller itself. These issues and how to properly address them will be discussed in the next section. 


\section{MVC Design Challenges: Control Effector Blending}

- Typical quadratic type formulations can result in controls "fighting" each other, specially if 2 control effectors have very similar influence on plant

- Use knowledge of problem physics to "blend" or "gang" control effectors into pseudo-controls

- Typically blend physical effectors into effective force and moment generators

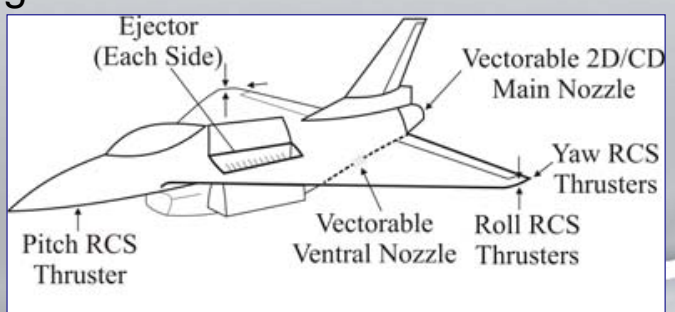

Glenn Research Center

at Lewis Field

\section{MVC Design Challenges: Control Effector Blending}

If the design plant is such that 2 or more control effectors have "similar" effect on the plant, then the "optimal" control law might be such that the control effectors end up "fighting" each other in order to provide the best performance. It is best to use the knowledge of the physics of the problem to blend or gang control effectors into pseudo-controls for the mathematical formulation. For physical control effectors, you would typically combine them into effective force and moment generators.

The schematic shown above is for a conceptual advanced STOVL (Short Take-Off and Vertical Landing) aircraft which was used for development of integrated flight/propulsion control methods (see Reference below). In the transition from cruise to hover phase, the aircraft is controlled using both aerodynamic effectors (elevons and rudder) and propulsive effectors (ejectors on the wings, vectorable main and ventral nozzle and 5 RCS (reaction control system) thrusters). Although there are 5 RCS thrusters, it is important to note that the Pitch RCS and the 2 Yaw RCS thrusters provide thrust only in one direction. So the Pitch RCS is used for pitch up commands while the two Roll RCS thrusters are used collectively for pitch down commands. Similarly, the right Yaw RCS is used for heading left whereas the left Yaw RCS is used for heading right. If both the Yaw RCS thrusters were used in the control design model, then it will result in a control law that uses the two thrusters differentially to improve yaw control which will be inconsistent with the implementation, i.e., only one or the other should be active. Similarly, if both the Roll RCS thrusters along with the Pitch RCS thrusters are used in the control design model, then the control law can result in Roll thrusters fighting the Pitch thruster and will also command downward Pitch RCS for pitch down maneuvers which is again inconsistent with the actual implementation. How to do the control blending to address the limitations of the RCS thrusters will be discussed next.

Ref: Garg, S., "Robust Integrated Flight/Propulsion Control Design for a STOVL Aircraft Using Hinfinity Control Design Techniques,” Automatica, vol. 29, no. 1, pp. 129-145, 1993. 


\section{MVC Design Challenges: Control Effector Blending (cont.)}

RCS blending for STOVL aircraft

- Use 3 "effective" RCS areas

- Allows formulating "design plant" such that it reflects how control effectors are actually used

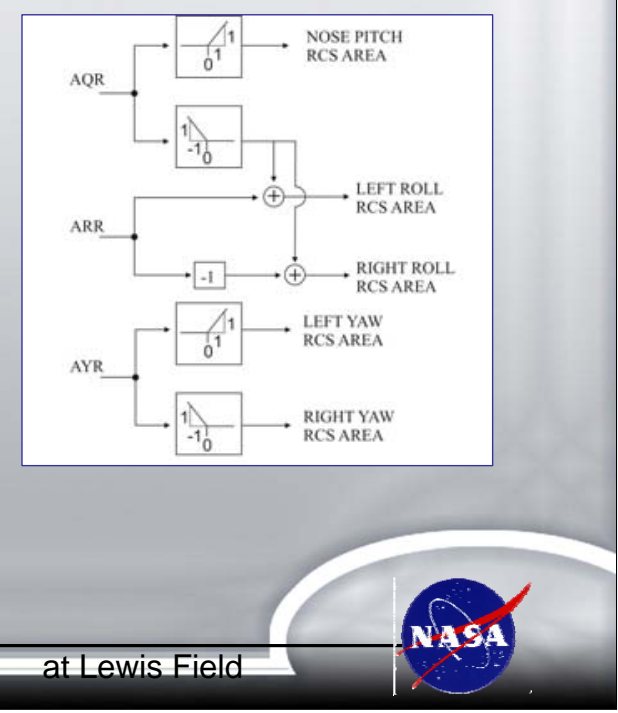

\section{MVC Design Challenges: Control Effector Blending (cont.)}

For the purposes of H-infinity based MVC synthesis for the STOVL aircraft, the 5 RCS thruster areas were combined into 3 effective areas - AQR, AYR and ARR for pitch, yaw and roll control, respectively. An RCS distribution logic that will distribute the three design model RCS commanded areas to the five actual areas in the nonlinear control implementation is shown in the figure above. Since the Pitch RCS thruster only provides positive (pitch up) pitching moment, a negative AQR command is distributed among the left and right wing tip RCS thrusters to generate the commanded negative pitching moment taking into account the relative pitch control effectiveness of the nose and wing tip RCS thrusters. The ARR command is distributed differentially among the 2 Roll thrusters, and the AYR command is sent to either the left or the right Yaw RCS thruster depending on the direction for the heading command.

This approach allows formulating the design plant such that it reflects how the control effectors are actually used. 


\section{MVC Design Challenges: Addressing Nonlinearities}

- Linear control design methods assume that "plant" behaves linearly in neighborhood of design point

- Not always true for some nonlinearities: e.g., Hysteresis, Stiction, Absolute value etc.

- For STOVL example:

- RCS uses bleed flow that acts as disturbance for engine

- WB Abs (ARCS) - you can't pump airflow into engine through RCS!

- Integrating airframe/engine model removes explicit dependence on bleed flow with ARCS appearing as direct control effectors

- Control design using linear integrated controller results in ARCS being used to improve engine dynamics by pumping flow back!

- Fix: Design model modified:

- Elements of "B" matrix from ARCS to engine states are zero

- Bleed flow added as external disturbance to engine states

Glenn Research Center at Lewis Field

\section{MVC Design Challenges: Addressing Nonlinearities}

The linear design methods assume that the plant behaves linearly in the neighborhood of the design point. This is not always true for some nonlinearities such as hysterisis, stiction, absolute value etc. Care should be taken in formulating the design plant when there are such nonlinearities in the system.

For the STOVL integrated flight/propulsion control design example, engine compressor bleed flow is used for the RCS thrusters. Note that there is an absolute value nonlinearity in the relationship from commanded RCS areas compressor bleed flow demand in that although the RCS area may be positive or negative, depending on the desired direction of the RCS thrust, the compressor bleed flow demand (WB) to generate the thrust is always positive. In an earlier control design problem formulation, this nonlinearity was not properly accounted for and the resulting controller was shown to lead to unacceptable performance when evaluated with this nonlinearity. The linear control design was such that RCS areas were being used to "pump back" air into the engine to regulate the engine dynamics in the presence of disturbances due to aircraft motion.

The physics of the problem says that the bleed flow acts as a disturbance on engine dynamics and that the RCS thrusters are to be used for flight control and not for engine control. To reflect this physics, the elements of the control input matrix, "B" in the linear design plant, which relate RCS areas to engine states were set to 0, i.e., the RCS areas do not affect the engine operating point, and bleed flow was added as an external disturbance affecting the engine dynamics in the design plant. This formulation resulted in a linear control law design which was able to regulate engine dynamics in the presence of bleed flow demands to achieve commanded aircraft maneuvers. See the Reference listed on page 8 for further details of this design plant formulation and the closed-loop controller evaluation. 


\section{MVC Design Challenges: Controller Stability}

- MVC design approaches "guarantee" closed-loop stability-what about stability of controller itself?

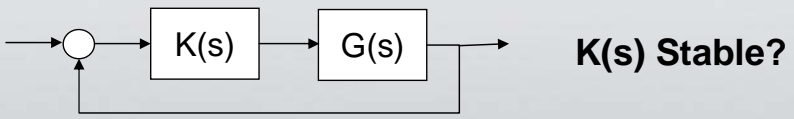

- Implementation issues such as independent $\mathrm{V} \& \mathrm{~V}$, operation across multiple modes, etc. require that controller implemented be stable

- Check resulting design from your favorite synthesis method to make sure controller is stable

- What if it is not?

- Do analysis to see if design plant is "strongly stabilizable"

- Try making changes in performance specifications and/or design plant formulation

\section{MVC Design Challenges: Controller Stability}

MVC synthesis techniques guarantee stability of the closed loop system, but what about the stability of the controller itself? None of these techniques guarantee the stability of the controller (K(s) in the above figure). The novice control designer might ask: "why do I care about controller stability as long as the closed-loop system is stable?”. One has to keep in mind that implementing the controller, as software in the control hardware, requires independent Verification and Validation (V\&V) of the control software. If the controller design is unstable, this V\&V becomes extremely difficult since the outputs will exceed the limits very fast. Also, typically there are multiple control modes for operation over a wide envelope. For aircraft, the control mode switches from ground control to flight control based on the WoW (Weight on Wheels) switch. Having an unstable controller in any of the modes makes it difficult to predict as to what is going to happen when the mode switching takes place.

Since MVC synthesis techniques do not guarantee controller stability, it is essential to check the stability of the controller resulting from a particular design formulation. If the controller is stable you can proceed to next step, otherwise you have to use trial and error to see if the synthesis problem can be formulated in such a way as to result in a stable controller. This might mean changing the performance specifications, i.e., choosing different weighting functions, adding different sensors, choosing different outputs to control etc. If the plant is unstable and you set aggressive performance goals, then there's a high likelihood that you might end up getting an unstable controller.

An unstable plant is called "strongly stabilizable" if there exists a stable controller which will stabilize the plant in a closed-loop system. If the plant is not strongly stabilizable, then no MVC approach will give a stable controller. However, most physical systems are "strongly stabilizable" or can be made so by the right choice of sensors. If repeated trials end up in an unstable controller, then it is worthwhile to perform analysis to see if the plant is strongly stabilizable. 


\section{MVC Implementation Challenges: Controller Scheduling}

- Implementation requires "gain scheduling" controller for operation over wide envelope

- Controller of order $n$ with $r$ inputs and $m$ outputs requires $n(1+m+r)+m r$ parameters scheduled

- Closed-loop stability not guaranteed between design points

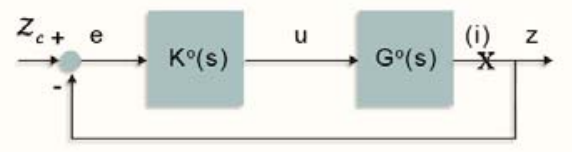

(a) Nominal Control Loop

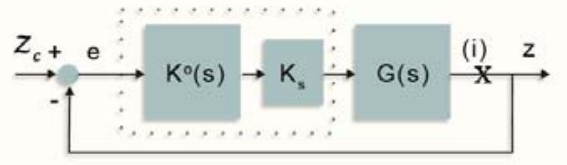

(b) Off-Design Control Loop
- Simplified scheme that exploits robustness of control design

- Match loop transfer function at controlled output

- Determine Ks to:

$$
\operatorname{Min} \| \mathrm{G}(\mathrm{s}) \mathrm{KsK}^{0}(\mathrm{~s})-
$$
$\mathrm{G}^{0}(\mathrm{~s}) \mathrm{K}^{0}(\mathrm{~s}) \|$

- Only $m^{2}$ parameters to schedule

\section{MVC Implementation Challenges: Controller Scheduling}

The traditional approach to designing feedback control laws for complex nonlinear systems is to design linear control laws at various operating points and then "gain schedule" the control laws through some kind of curve fitting of the various controller gains with the critical operating point variables as the independent scheduling parameters. One of the difficulties with implementing gain scheduling for MVC laws is the complexity of the scheduling. For a controller of order $n$ with $r$ inputs and $m$ outputs, there will be $n(1+m+r)+m r$ controller parameters to schedule. Furthermore, although the closed-loop system is stable for all design points, the stability of the closed-loop system is not guaranteed when operating between design points.

The fundamental objective in using Robust MVC design techniques is to reduce the controller complexity while guaranteeing the desired performance and robustness characteristics. The above figure shows a simplified controller scheduling scheme which consists of scheduling only the output matrix of a nominal controller which is designed to give a robustly stable closed-loop system over a wide range of plant operating conditions. The scheduling scheme is of the form: $\mathrm{K}(\mathrm{s})=\mathrm{KsK}^{0}(\mathrm{~s})$, where $\mathrm{K}(\mathrm{s})$ is the scheduled controller, Ks is the controller output scheduling matrix and $\mathrm{K}^{0}(\mathrm{~s})$ is the nominal controller. For such a scheduling scheme there are only $\mathrm{m}^{2}$ parameters to schedule. The approach to synthesize the scheduling gains Ks consists of applying parameter optimization techniques such that the transfer function matrix with the loop broken at the controlled outputs ( $(i)$ in fig. b. above) "closely" matches the output loop transfer matrix of the nominal control loop. The details of the controller scheduling synthesis approach are described in the Reference listed below. This scheduling scheme is highly effective when the major change in the plant across the different operating points is in control effectiveness. An example application will be discussed next.

Ref.: Garg, S., “A Simplified Scheme for Scheduling Multivariable Controllers,” IEEE Control Systems, August 1997, pp. 24-30. 


\section{MVC Implementation Challenges: Controller Scheduling (cont.)}

- Solved as constrained parameter optimization problem:

- Control design at 80 knots provided stability robustness for 100-knot model

- Primary change from 80to $100-$ knot model was in control effectiveness

- Simplified scheduling scheme provided close to "nominal" response at off-design point

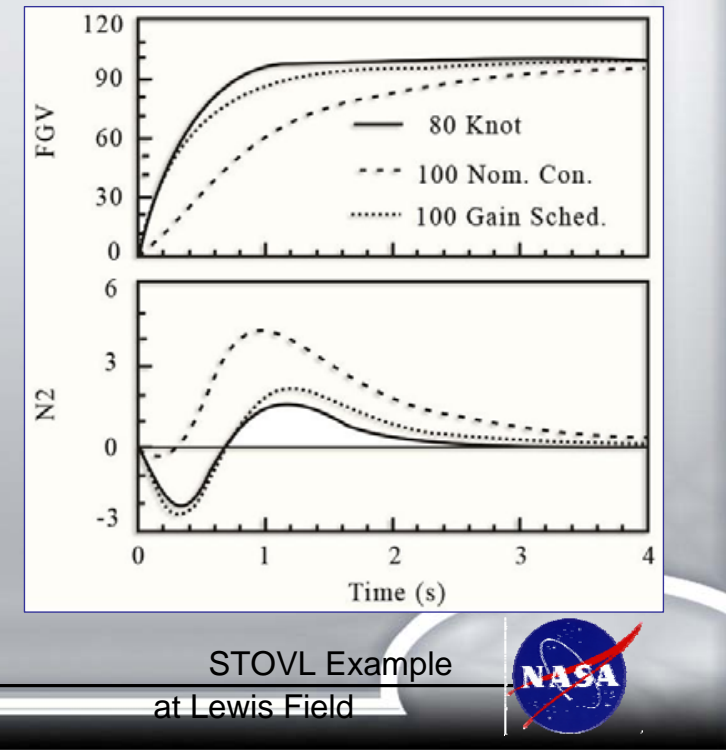

\section{MVC Implementation Challenges: Controller Scheduling (cont.)}

The STOVL IFPC design discussed earlier was implemented as separate, but interconnected, flight and propulsion controllers. The $\mathrm{H}$-infinity based design was performed at the nominal flight condition corresponding to 80 knots air speed. The controller was designed for the transition flight regime, where the control of the aircraft is transitioning from aerodynamically generated forces to propulsive forces, which ranges from 50 to 120 knots. Since the nominal control design provided closed loop stable response throughout the transition flight regime, and also the most significant change in aircraft response to control inputs in this operating regime is that of control effectiveness, it was decided to apply the simplified controller scheme to extend the operation of the nominal control design to the transition flight regime.

The results for the controller scheduling scheme application to the propulsion control are shown in the above figure. The solid line in the figure shows the response of the propulsion system to a step command in the Ventral nozzle thrust (FGV) for the nominal design point with the nominal controller. The dashed line shows this response for the propulsion model at 100 knots with the nominal controller. The need for scheduling the controller is evident from the degraded FGV command tracking response and the increased coupling with the engine rotor speed (N2). The dotted line shows the response when the controller is scheduled using the gains from the simplified scheduling scheme. The results indicate that the simplified scheduling provides a response which closely matches the response of the nominal system with the nominal controller in decoupled command tracking.

Detailed results of the application of the simplified scheduling scheme to the STOVL propulsion system control design are described in the reference listed on page 12, and those for the IFPC design are described in the reference listed below.

Ref.: Garg, S., and Mattern, D., “Application of an Integrated Methodology for Propulsion and Airframe Control Design to a STOVL Aircraft,” AIAA Paper no. 94-3611, Guidance, Navigation and Control Conference, Scottsdale, AZ, August 1994. 


\section{MVC Implementation Challenges: Integrator Windup Protection}

- What is integrator windup?

- Integrator action in controller drives steady-state errors to zero

- If controller outputs are limited, such as actuator position limit, integral action will continue to command higher controller outputthus controller integrator(s) will wind up

- Results in deteriorated performance since controller integrator must first "unwind" when high actuator deflections are not needed

- Windup can even lead to system instability

- IWP Design Requirements:

- Should: be memory-less; provide smooth transfer; be closed-loop stable; provide graceful performance degradation

- For SISO, IWP simply requires "stopping" integration when limit is encountered

- How to do this in MVC systems?

Glenn Research Center at Lewis Field NASA

\section{MVC Implementation Challenges: Integrator Windup Protection (IWP)}

In a control system, the effort to drive steady-state errors to zero is accounted for through integral action on part of the controller. If at any point in time the controller outputs are limited and controller inputs are non-zero and of the same numerical sign (+/-), the integral action of the controller will attempt to increase the magnitude of the already limited controller output, thus the controller's integrator(s) will wind up. Following this period of integrator windup, the controller response to command inputs might be very poor because the controller's integrators must first unwind prior to attempting to calculate the new controller outputs for the new controller inputs. Thus, Integrator Windup Protection (IWP) must be included in control systems designed for tracking commands with zero steady-state error.

Integrator windup protection (IWP) design requirements are: i) Should be memory-less and not contribute to controller output when actuation system limits are not encountered; 2) Should provide smooth transfer between limited and unlimited case and provide accurate tracking of limited actuator; 3) Should be closed-loop stable for all possible limit combinations; 4) Should attempt to maintain system performance and provide graceful degradation when limit is encountered.

For single-input single-output systems, simply stopping the integration when a limit is encountered meets all the IWP requirements. It is not clear as to how to best implement IWP in multivariable control systems. Numerous ideas for integrator windup protection and bumpless transfer have been published in literature and will be discussed briefly next. 


\section{MVC Implementation Challenges: IWP (cont.)}

- Controller states modified based on difference between commanded and limited actuator value

- Gains selected to provide stable effective controller, 1 column at time for single limited actuator

- Typical IWP synthesis techniques require controller to be represented in specific form and do not guarantee closed-loop system stability

- Very much trial and errc

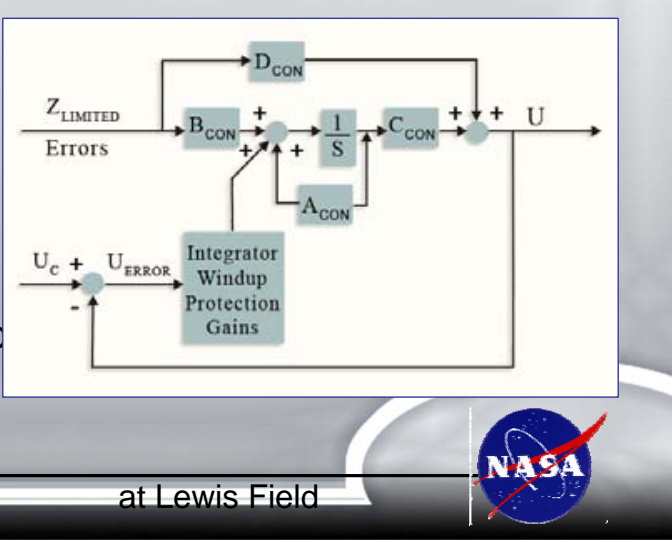

Glenn Research Center

\section{MVC Implementation Challenges: IWP (cont.)}

To implement IWP on MVC systems, the controller is represented in the form shown in the above figure. The block "Integrator Windup Protection Gains" is added to the controller to modify the controller states whenever an actuation limit is encountered. The inputs to this block are the errors between the outputs of the controller (the commands to the actuators) and the actual actuator positions with the limit in place. This implementation of the controller meets IWP design requirement 1 , that the IWP be memoryless and not affect the system when the controller outputs are not being limited.

The IWP design techniques for MVC systems that have been published in literature consist of selecting the IWP gains one column at a time considering a single actuator to be limited. The column of gains is selected to ensure that the modified controller remains stable and provides good tracking of the limited command so as to minimize integrator windup, thus meeting the IWP requirement 2 listed earlier. However, this approach does not guarantee that the closed loop system will remain stable when the IWP becomes active. Neither does it provide a systematic way of ensuring that the closed-loop performance will degrade gracefully when the limits are encountered. Application of this approach to the STOVL IFPC design resulted mostly in severe degradation in performance when the actuator limits were encountered, and in some instances this approach even resulted in the closedloop system being unstable with the modified controller.

A parameter optimization based scheme was developed to determine the IWP column gains such that for a single actuator saturation, the modified controller and the closed-loop system will remain stable while minimizing the loss in tracking performance compared to the "unlimited" system performance. This approach, which is described in detail in reference listed below, will be discussed next.

Ref.: Watts, S.R., and Garg, S., “An Optimized Integrator Windup Protection Technique Applied to a Turbofan Engine Control,” AIAA Paper No. 96-3814, Guidance, Navigation and Control Conference, San Diego, CA, July 1996. 


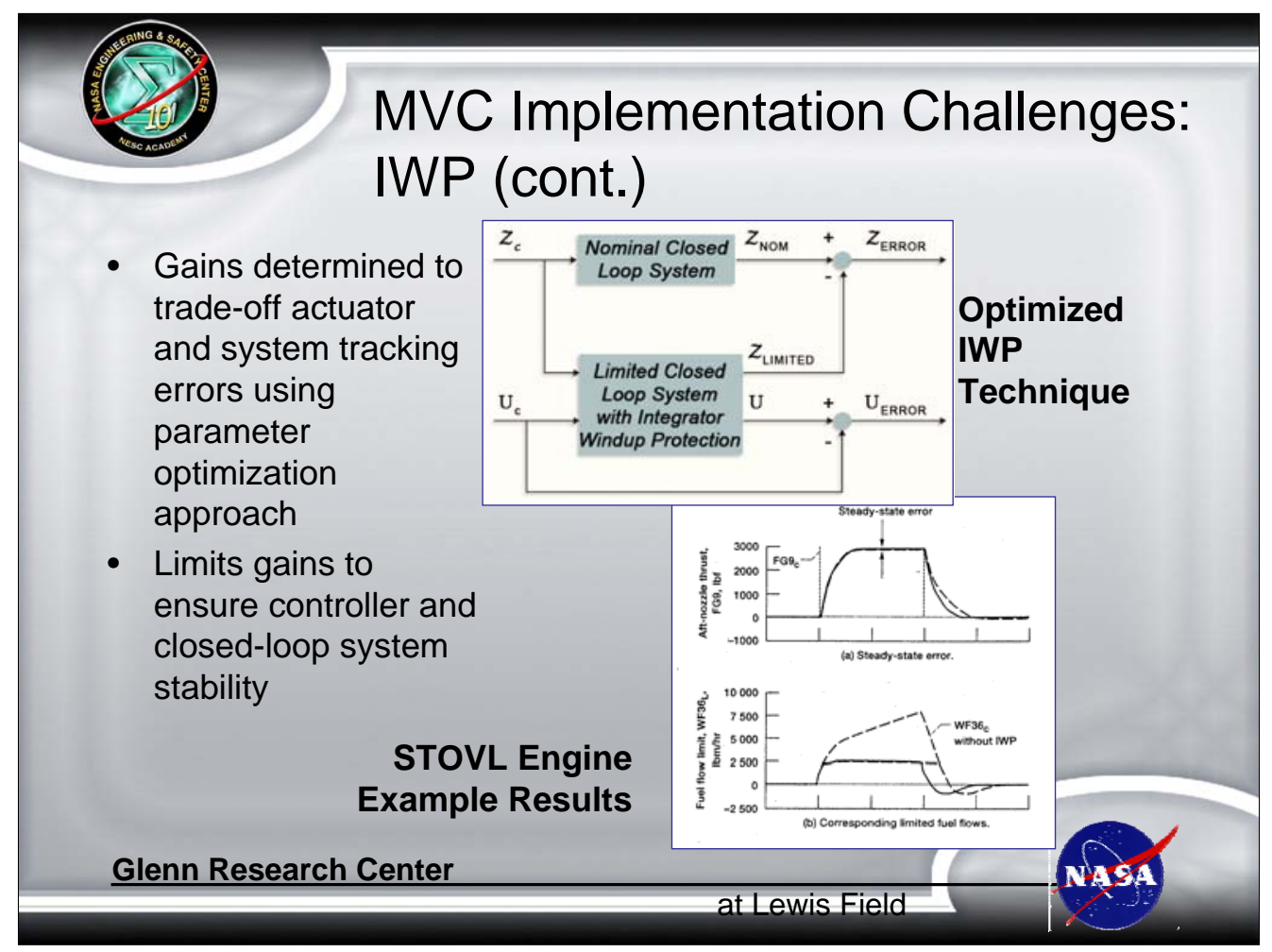

\section{MVC Implementation Challenges: IWP (cont.)}

The generalized design plant for determining the parameter optimization based IWP gains is shown in the figure above. This problem set up allows for the calculation of errors between the nominal closed-loop, or "ideal" system, and the limited system with IWP for the performance loops and the actuator command tracking error. The performance errors, ZERROR, are defined as the difference between the response of controlled variables for the nominal system, ZNOM, and the response of the controlled variables for the limited system with IWP, ZLIMITED. The controlled actuator error, UERROR, is defined as the difference between the commanded actuator position, UC, and the controller with IWP commanded actuator position, U. For a single actuator being limited, the corresponding column of the IWP gains is obtained by minimizing the weighted sum of squares of ZERROR and $U$. The relative weighting between these two errors can be used as a "design handle" to trade-off between providing tight IWP versus providing tighter tracking performance. The closedloop stability and controller stability is guaranteed with the selected IWP gains by checking for both during the optimization process and setting the cost being minimized to be high if any of these conditions is violated.

The second figure above, from reference listed below, shows an example result from application of the optimized IWP technique to the propulsion control portion of the STOVL IFPC design. Shown are the response of the aft nozzle thrust (FG9) to a step command and the corresponding fuel flow (WF36) requirement with and without the IWP. Without IWP, dashed lines, when the fuel flow limit is encountered, the fuel flow command continues to grow because of integrator wind up. As the FG9 command is taken out, the integrator takes time to wind down and the FG9 response is degraded. With IWP active, solid lines, the fuel flow command tracks the fuel flow limit and good FG9 tracking performance is obtained when the step command is removed.

Ref.: Mattern, D., and Garg, S., "Propulsion System Performance Resulting from an Integrated Flight/Propulsion Control Design,” AIAA Paper No. 1992-4602, Guidance, Navigation and Control Conference, Hilton Head, SC, August 1992. 


\section{Iterative Process for Control Design}

- Simplistic look at control design and implementation process:

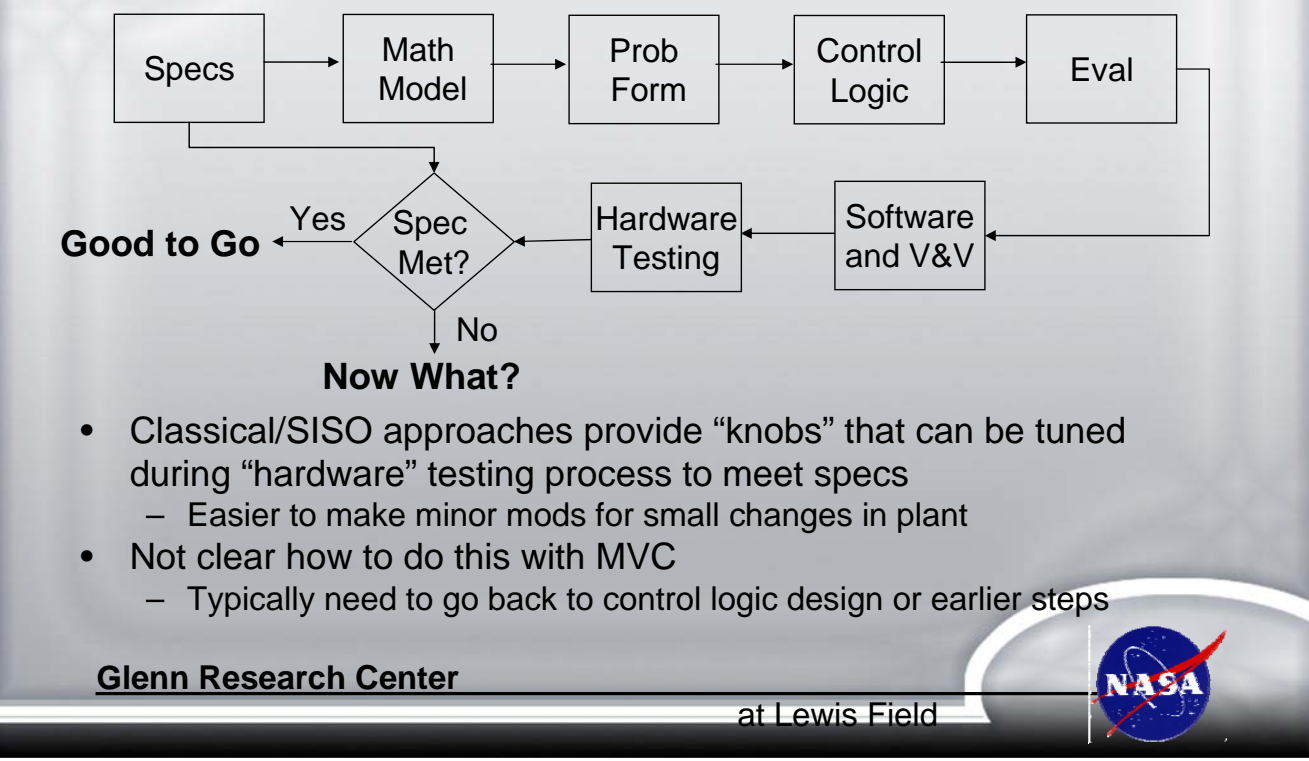

\section{Iterative Process for Control Design}

The figure above provides a very simplistic look at the control design process. Given performance specifications and a math model of the system, the control design problem is formulated in such a way as to provide the desired trade-off between performance, robustness and control usage. Once an acceptable control law is obtained through the control synthesis process, it is implemented in the nonlinear simulation and tested to ensure that controller scheduling and limit protection are appropriately implemented. Once the nonlinear evaluation provides satisfactory results, then the control laws are converted to software to be implemented in the control hardware. Extensive software V\&V (Verification and Validation) is conducted to ensure that the software is error free and faithfully implements the control law as designed. The control software is then implemented on hardware and hardware-in-loop testing and testing on the actual system (flight test for example) is conducted.

This is where the so-called "rubber hits the road". Till now all the control development and evaluation had been based on some mathematical models, and mathematical formulations of uncertainties etc. Once the control is implemented on the real system and tested under realistic operating conditions, that is the final test of whether the control design meets the system performance objectives or not. Rarely does it happen that the control design as first implemented meets all the objectives over the wide operating envelope. Typically there will be shortcomings identified in the testing and these will have to be fixed in order to certify the control design as fully operational.

For single-input single-output systems with classical control structure, such as PID (Proportional+Integral+Derivative), the control gains provide individual "tuners" to be adjusted during testing to make modifications as needed. How to do such modifications on the fly for MVC systems is not at all clear. One almost has to go back to the step one of reformulating and resynthesizing the original control law in order to address the shortcoming. This is one of the fundamental reasons why MVC has not found application in operating aerospace systems. 


\section{Modifying MVC Designs}

- Understanding physics of problem useful to make mods without having to resort to major control redesign

- STOVL example:

- Initial piloted evaluation showed high-frequency pitch oscillation

- Cause of oscillation isolated to time-delays because of digital implementation; duplicated by including time delay in simulation

- Pitch damping improved by adding pitch rate error feedback to elevator
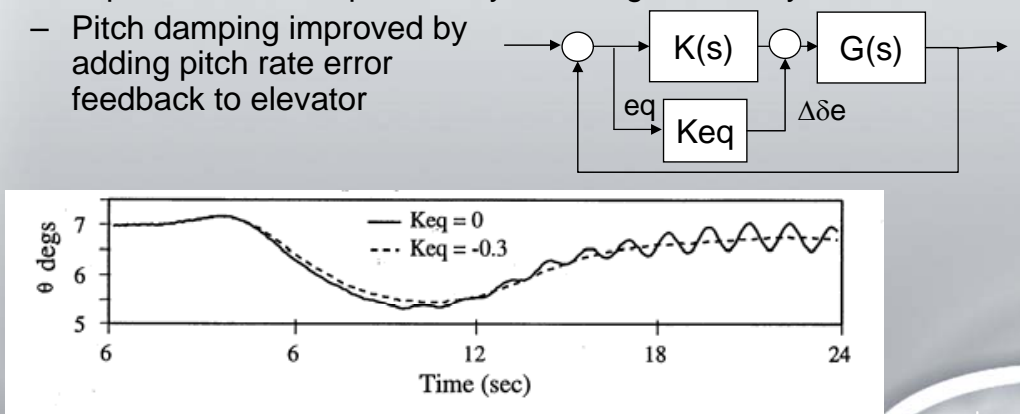

Glenn Research Center

\section{Modifying MVC Designs}

All is not lost though when it comes to having to make modifications to the MVC design in the final stages of testing. Understanding the physics of the problem comes in useful in making the modifications without having to go back to step 1 of reformulating the control design problem and starting all over with the controller synthesis.

For the STOVL IFPC design, initial piloted evaluations in a fixed-base simulator uncovered some problems which had not been apparent in the non-realtime evaluation of the design. One of the problems was a pitch oscillation during deceleration from cruise to hover. The figure above shows the pitch attitude response of the aircraft, from the simulator, for a simultaneous command of flight path change and deceleration. The solid line in the figure above is for the nominal IFPC design and shows the pitch oscillation. Analysis indicated that this oscillation is due to the significant time delay between the computer that hosts the aircraft simulation and the computer that implements the IFPC design. The IFPC design was such that it resulted in a low damped aircraft short period mode, and the phase loss due to time delay in the real-time simulation environment resulted in the observed oscillatory behavior.

To improve the damping of the pitch mode, the IFPC design was modified to include an additional constant gain feedback loop from the pitch rate error to the elevator as shown in the block diagram above. The root locus technique was used to determine the value of the feedback gain to provide improved short period mode damping without having any significant effect on the other closed-loop poles. The dashed line in the above figure shows that with this modification, the IFPC design resulted in well damped pitch response. The reference listed below provides additional details of how such knowledge of the physics led to successful implementation of the IFPC design.

Ref: Bright, M.M., et al. "Piloted Evaluation of an Integrated Methodology for Propulsion and Airframe Control Design,” AIAA Paper No. 94-3612, Guidance, Navigation and Control Conference, Scottsdale, AZ, August 1994. 


\section{Conclusion}

- Potential benefit of multivariable control design:

"Improved performance and robustness to modeling uncertainties achievable with highly complex systems"

- Use MVC design with Caution:

- Make sure MVC is really needed

- Get good understanding of physics of problem

- You cannot make 747s fly like F-16s, no matter how good a control engineer you are!

- Make sure that synthesis problem is formulated right

- Control effector blending

- Nonlinearities around small perturbation model

- Controller stability and order

- Give thought to scheduling and IWP issues before deciding on control structure

- Keep these as simple as possible

- Use knowledge of system to make "smart" amendments based on test results

Glenn Research Center at Lewis Field Nas

\section{Conclusion}

Potential benefit of multivariable control is the improved performance and robustness to modeling uncertainties, achievable with highly complex systems. So if you are working with a highly complex system and very tight performance requirements, then maybe multivariable control is the way to go. However, multivariable control should be used with caution and analysis should be done to ensure that the use of MVC is justified.

As discussed throughout the paper, getting a good understanding of the physics of the problem is essential for the MVC design to be successful in realistic application. Remember that the control synthesis process is just a convenient mathematical formulation which results in a tractable, solvable control law. The formulation has to take into account the inherent limitations of achievable performance for the "plant". You cannot make a 747 fly like an F-16, no matter how good a control engineer you are. Formulating the synthesis problem right means you pay adequate attention to issues such as control effector blending, available control authority and effect of nonlinearities around the small perturbation model. Once the synthesis process results in a controller that meets the closed-loop performance specifications, at least for the design plant, then from an implementation perspective, it is important to ensure that the controller itself is stable and that its order can be reduced to a "reasonable" number of states. Before starting with the controller synthesis process, thought should be given to the overall controller structure in terms of gain scheduling and integrator wind up protection. As has been discussed in the paper, the robustness characteristics of the controller design can be used to simplify the controller scheduling.

Finally, use knowledge of the system to make smart amendments based on test results. Keep in mind that the controller will be implemented in discrete form and computational effort and sampling will result in effective time delays which should be accounted for in the design. Some of the shortcomings of the MVC design can be overcome in the implementation stage by applying classical control methods in conjunction with a good understanding of the plant dynamics. 


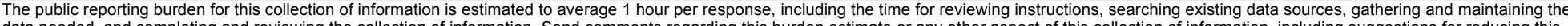

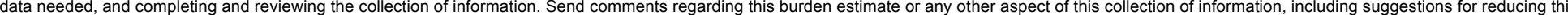

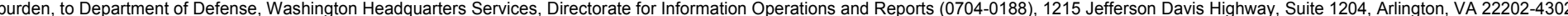

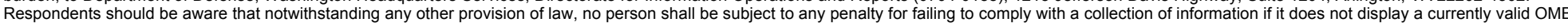
control number.

PLEASE DO NOT RETURN YOUR FORM TO THE ABOVE ADDRESS.

\section{REPORT DATE (DD-MM-YYYY) \\ 2. REPORT TYPE \\ 3. DATES COVERED (From - To)}

01-01-2008

\section{TITLE AND SUBTITLE}

Technical Memorandum

Implementation Challenges for Multivariable Control

What You Did Not Learn in School!

\section{5a. CONTRACT NUMBER}

5b. GRANT NUMBER

5c. PROGRAM ELEMENT NUMBER

\section{AUTHOR(S)}

Garg, Sanjay

\section{5d. PROJECT NUMBER}

5e. TASK NUMBER

5f. WORK UNIT NUMBER

WBS 732759.03.01.02.17

\section{PERFORMING ORGANIZATION NAME(S) AND ADDRESS(ES)}

National Aeronautics and Space Administration

\section{PERFORMING ORGANIZATION REPORT NUMBER}

John H. Glenn Research Center at Lewis Field E-16220

Cleveland, Ohio 44135-3191

\section{SPONSORING/MONITORING AGENCY NAME(S) AND ADDRESS(ES)}

National Aeronautics and Space Administration

Washington, DC 20546-0001

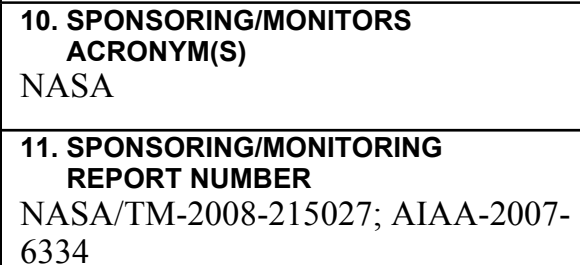

\section{DISTRIBUTION/AVAILABILITY STATEMENT}

Unclassified-Unlimited

Subject Category: 31

Available electronically at http://gltrs.grc.nasa.gov

This publication is available from the NASA Center for AeroSpace Information, 301-621-0390

\section{SUPPLEMENTARY NOTES}

\section{ABSTRACT}

Multivariable control allows controller designs that can provide decoupled command tracking and robust performance in the presence of modeling uncertainties. Although the last two decades have seen extensive development of multivariable control theory and example applications to complex systems in software/hardware simulations, there are no "production" flying systems--aircraft or spacecraft, that use multivariable control. This is because of the tremendous challenges associated with implementation of such multivariable control designs. Unfortunately, the curriculum in schools does not provide sufficient time to be able to provide an exposure to the students in such implementation challenges. The objective of this paper is to share the lessons learned by a practitioner of multivariable control in the process of applying some of the modern control theory to the Integrated Flight Propulsion Control (IFPC) design for an advanced Short Take-Off Vertical Landing (STOVL) aircraft simulation.

15. SUBJECT TERMS

Engine control; Health management; Adaptive control; Intelligent systems; Fault diagnostics

\section{SECURITY CLASSIFICATION OF:}

a. REPORT b. ABSTRACT

\section{LIMITATION OF} ABSTRACT

$\mathrm{UU}$
18. NUMBER

OF PAGES 25 19a. NAME OF RESPONSIBLE PERSON STI Help Desk (email:help@sti.nasa.gov) 19b. TELEPHONE NUMBER (include area code) 301-621-0390 

